\title{
Analysis of Pb-Bi Vessel Wall Ablation Experiment with High Temperature Liquid by MPS Method
}

\author{
Daisuke Masumura $^{1}$, Akifumi Yamaji ${ }^{1}$ and Masahiro Furuya ${ }^{2}$ \\ 1. Cooperative Major in Nuclear Energy, Graduate School of Advanced Science and Engineering, Faculty of Science and \\ Engineering, Waseda University, Tokyo 169-8555, Japan \\ 2. Nuclear Technology Research Laboratory, Central Research Institute of Electric Power Industry, Tokyo 201-8511, Japan
}

Received: September 15, 2015 / Accepted: October 09, 2015 / Published: November 30, 2015.

\begin{abstract}
In a severe accident of a light water reactor, ablation of the RPV (reactor pressure vessel) lower head by corium is a key phenomenon, which affects progression of the accident. The MPS (moving particle semi-implicit) method is one of particle methods that calculate behavior of incompressible fluid by semi-implicit method. In preceding studies, the MPS method has been extensively studied and developed for simulations of different phenomena involved in severe accident of nuclear reactors. This paper aims to investigate whether the MPS method is capable of analyzing the lower head ablation phenomenon. The small-scale experiment carried out at CRIEPI (Central Research Institute of Electric Power Industry) using Pb-Bi vessel and silicone oil was analyzed for the validation of the MPS method. The MPS analysis well reproduced the experimental phenomena qualitatively. However, with respect to some quantitative results, more investigation such as influence of the calculation particle size is necessary.
\end{abstract}

Key words: Severe accident, ablation, particle method, the MPS method, stratification.

\section{Introduction}

In a severe accident of a LWR (light water reactor), the core may melt down and drop to the bottom of a RPV (reactor pressure vessel). Due to decay heat of the molten core materials (corium) and debris, the RPV lower head may suffer intensive thermal attack, which may ablate the vessel wall [1]. Consequently, thinning of the vessel wall and other structural materials may lead to failure of the vessel. Ablation of the vessel wall is also a key phenomenon, which determines validity of IVR (in-vessel retention) of corium [2]. Hence, accurate modeling and prediction of ablation is essential for understanding severe accident progression as well as for development of IVR concepts. However, despite its importance, there is limited experimental knowledge about RPV lower head failures, because such experiments are highly expensive and also

\footnotetext{
Corresponding author: Daisuke Masumura, student, research field: severe accident. E-mail: 0710masumura@toki.waseda.jp.
}

difficult to conduct. Moreover, these experiments often focused on creep rupture of the vessel under high pressure as they were mainly intended for severe accidents of PWRs (pressurized water reactors) [3, 4]. In BWR (boiling water reactor) severe accidents, ablation of the RPV lower head may be more important as the reactor system is designed to depressurize at the early stage of an accident.

Different severe accident codes have been developed, and used to predict failures of RPVs in the Fukushima Daiichi Nuclear Power Plant Accident (March, 2011). However, there seems to be large uncertainties among different codes (e.g., MELCOR, MAAP and SAMPSON) in predictions of RPV lower head failures [5-7]. In these system analysis codes, where phenomena associated with lower head failure are poorly understood, such as penetration failure, the models are very simple and parametric. Moreover, the lower head nodalizations are often simple and cannot simulate stratification of corium accurately and hence, 
cannot simulate accurate heat flux distribution from corium to the vessel wall accurately, which leads to inaccurate prediction of the vessel wall ablation [8]. Adoptions of simple models and correlations in these codes arise from the nature of Eulerian methods, in which liquid flow and pressure need to be calculated for each calculation mesh.

In contrast, Lagrangian methods are much more suitable for simulations of the complex phenomena at the lower head, because flow and pressure of liquid can be modeled by motion and interactions of the calculated points (particles), which enable accurate simulation of convection, stratification, phase change and heat transfer based on physical models (rather than empirical correlations). Accurate modeling of these phenomena based on physical models is important for analyzing the lower head ablation accurately.

MPS (moving particle semi-implicit) method is one of the particle methods for incompressible flow and is based on a Lagrangian method [9]. The MPS method has been extensively studied and developed for simulations of different phenomena involved in severe accident of nuclear reactors [10-12]. Through these studies, it has been shown that, MPS is capable of accurately analyzing phase changes (solidification, melting, and ablation of metals), convection, stratification and heat transfer $[13,14]$. It can be expected that, based on these validated models, MPS will be able to accurately analyze ablation of RPV lower head for simulation of severe accidents. The aim of this study is to investigate whether the MPS method is capable of analyzing the lower head ablation phenomenon, which involves complex interactions of the above mentioned phenomena.

For the validation purpose of the MPS method, a small-scale experiment was carried out at CRIEPI (Central Research Institute of Electric Power Industry) using $\mathrm{Pb}-\mathrm{Bi}$ as a vessel and silicone oil as the confined liquid which ablates the vessel wall [15]. Since the MPS method does not rely on empirical correlations which are dependent on geometrical scale and the models are developed based on physical models rather than empirical models, it is expected that, simulation of the small-scale experiment will provide valuable information regarding validity of the models and identify areas where further study and development may be necessary to analyze RPV lower head ablation.

\section{Calculation Method}

\subsection{MPS Method}

The MPS method is one of particle methods that calculate behavior of incompressible fluid by semi-implicit method. The term "semi-implicit" method indicates that, viscosity and gravity are calculated by explicit method while pressure is calculated by implicit method. Particle method is based on Lagrangian method and expresses continua like fluid by particles. It is suitable for analysis of fluid flow involving phase changes and large deformations, because it is capable of accurately analyzing motions of free surfaces by tracking motions and interactions of the particles. In contrast, Eulerian method exhibits some difficulties with simulating such phenomena. For example, FEMs (finite element methods) have difficulties with large distortions of meshes and VOF (volume of fluid) methods tend to encounter numerical divergence at the boundary of free surface when simulating large deformation of free surface.

The MPS method used in this study is based on MPS-SW-MAIN-Ver2.0 developed by Koshizuka and Shibata [9]. Governing equations are mass, momentum, energy conservations as follows, respectively:

$$
\begin{gathered}
\frac{D \rho}{D t}=0 \\
\frac{D u}{D t}=-\frac{1}{\rho} \nabla P+v \nabla^{2} u+g \\
\frac{D h}{D t}=k \nabla^{2} T+Q
\end{gathered}
$$

where, $\rho$ is density, $u$ is velocity, $P$ is pressure, $v$ is kinematic viscosity, $g$ is gravitational acceleration, $h$ 
is enthalpy, $k$ is thermal conductivity, $Q$ is heat source.

In the MPS method, particle interaction models which express differential operators such as gradient, divergence and Laplacian are used for discretizing differential equations. These models are applied for neighboring particles by the weight function as:

$$
\omega(r)=\left\{\begin{aligned}
\frac{r_{e}}{r}-1 & \left(0 \leq r \leq r_{e}\right) \\
0 & \left(r_{e} \leq r\right)
\end{aligned}\right.
$$

where, $r$ is distance between particles, $r_{e}$ is radius of influence that determines neighboring particles. Particle interactions occur when $r$ is smaller than $r_{e}$.

The particle number density is expressed as summation of weight functions:

$$
n_{i}=\sum_{j \neq i} \omega\left(\left|r_{j}-r_{i}\right|\right)
$$

The MPS method is applied to incompressible fluid so that the particle number density is kept constant at the initial value $\left(n^{0}\right)$ throughout the analysis.

The gradient, divergence and Laplacian operators in the governing equations are expressed with the particle interaction model as follows, respectively:

$$
\begin{gathered}
\langle\nabla \phi\rangle_{i}=\frac{d}{n^{0}} \sum_{j \neq i}\left[\frac{\phi_{j}-\phi_{i}}{\left|r_{j}-r_{i}\right|^{2}}\left(r_{j}-r_{i}\right) \omega\left(\left|r_{j}-r_{i}\right|\right)\right] \\
\langle\nabla \cdot u\rangle=\frac{d}{n^{0}} \sum_{j \neq i} \frac{\left(u_{j}-u_{i}\right) \cdot\left(r_{j}-r_{i}\right)}{\left|r_{j}-r_{i}\right|^{2}} \omega\left(\left|r_{j}-r_{i}\right|\right) \\
\left\langle\nabla^{2} \phi\right\rangle_{i}=\frac{2 d}{\lambda n^{0}} \sum_{j \neq i}\left[\left(\phi_{j}-\phi_{i}\right) \omega\left(\left|r_{j}-r_{i}\right|\right)\right]
\end{gathered}
$$

where, $d$ is number of dimension in the analysis and $\lambda$ is the parameter that fit increase of statistical dispersion with analytical solution.

\subsection{Heat Transfer, Convection and Phase Change Models}

In the MPS method, when heat transfer between two different materials is calculated, thermal conductivity is expressed by a harmonic average of each one $[10,16]$ as shown in Eq. (9):

$$
k=\frac{2 k_{i} k_{j}}{k_{i}+k_{j}}
$$

where, $k_{i}$ and $k_{j}$ are thermal conductivities of material $i$ and $j$, respectively. However, in case of two different materials whose physical properties are quite different like this study, this average model may underestimate thermal conductivity. Hence, in this study, following arithmetic mean model is proposed to model a sharp change in thermal conductivity from one material to the other.

$$
k=\left(\sqrt{\frac{T_{i}}{T_{j}}}\right) \frac{2 k_{i} k_{j}}{k_{i}+k_{j}}+\left(1-\sqrt{\frac{T_{i}}{T_{j}}}\right) \frac{k_{i}+k_{j}}{2} \quad\left(T_{i} \leq T_{j}\right)
$$

where, $T_{i}$ and $T_{j}$ are temperature of material $i$ and $j$, respectively.

Radiation heat transfer is expressed by Stefan-Boltzmann's law as follows [16]:

$$
q=\varepsilon \sigma T^{4}
$$

where, $q$ is heat flux, $\varepsilon$ is emissivity, $\sigma$ is Stefan-Boltzmann constant. It is applied to a surface of liquid and the vessel wall.

Buoyancy by temperature difference is expressed by Boussinesq approximation as follows [16]:

$$
\frac{D u}{D t}=-\frac{1}{\rho} \nabla P+v \nabla^{2} u+g \beta_{0}\left(T-T_{0}\right)
$$

where, $\beta_{0}$ is coefficient of thermal expansion. Natural convection is reproduced by this approximation in the analysis.

Phase change is considered between solid phase and liquid phase. It depends on enthalpy of each particle. When enthalpy of a solid particle is larger than certain value, that particle becomes a liquid one and will be able to move according to the basic governing equations described in Section 2.1. On the other hand, when enthalpy of a liquid particle is smaller than certain value, that particle becomes a solid one $[10,16]$. Therefore, temperature of particles is determined as follows:

$$
T=\left\{\begin{array}{c}
T_{m}+\frac{h-h_{0}}{\rho C_{s}}\left(h<h_{0}\right) \\
T_{m}\left(h_{0} \leq h \leq h_{1}\right) \\
T_{m}+\frac{h-h_{1}}{\rho C_{l}}\left(h_{1}<h\right)
\end{array}\right.
$$

where, $T_{m}$ is melting point, $h_{0}$ and $h_{1}$ are enthalpies at melting start and end points, respectively. $C_{s}$ and $C_{l}$ are specific heat of solid and liquid state, respectively. 


\section{Analysis of the Experiment}

\subsection{Experimental Conditions}

As is explained in Chapter 1, the purpose of this study is to validate MPS method against an experiment, which captures the governing phenomena involved in ablation of RPV of a nuclear reactor, such as convection, stratification, phase change and heat transfer. As described in Chapter 2, the MPS models corresponding to these phenomena do not rely on empirical correlations and do not depend on scale of the calculation domain. These models are also independent of the materials. In another word, the same model can be used while differences due to material properties can be incorporated by replacing the relevant material parameters (e.g., thermal conductivity, thermal expansion coefficient, specific heat capacity).

Hence, a small-scale experiment, in which high temperature silicone oil was filled into the hemispherical vessel of $\mathrm{Pb}-\mathrm{Bi}$ was conducted by CRIEPI. Fig. 1 shows the cross-sectional view of the hemispherical vessel of $\mathrm{Pb}-\mathrm{Bi}$ and Table 1 shows the experimental condition.

The characteristic of the experiment is as follows: (1) At the beginning of the experiment, silicone oil with initial temperature of $300{ }^{\circ} \mathrm{C}$ was filled into the vessel with ambient temperature of $26^{\circ} \mathrm{C}$ from the top of the vessel. Filling of the vessel with the entire silicone oil of $80.7 \mathrm{~g}$ was completed in $1.63 \mathrm{~s}$. In the meantime, some of the oil was discharged from the

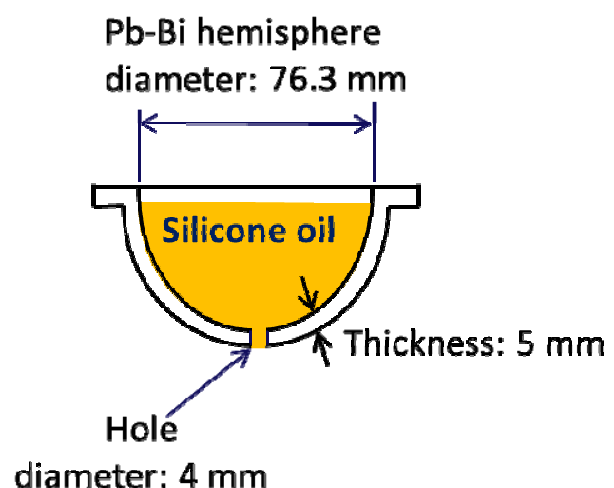

Fig. 1 Cross-sectional schematic of the hemispherical vessel.
Table 1 Experimental conditions.

\begin{tabular}{ll}
\hline Liquid & Silicone oil \\
\hline Initial temperature $\left({ }^{\circ} \mathrm{C}\right)$ & 300 \\
Kinematic viscosity coefficient $\left(\mathrm{mm}^{2} / \mathrm{s}\right)$ & $200\left(\right.$ at $\left.25^{\circ} \mathrm{C}\right)$ \\
Ambient temperature $\left({ }^{\circ} \mathrm{C}\right)$ & 26 \\
Filled mass of silicone oil $(\mathrm{g})$ & 80.7 \\
Filled time (s) & 1.63 \\
\hline
\end{tabular}

bottom hole; (2) The internal vessel wall of $\mathrm{Pb}-\mathrm{Bi}$ was ablated by silicone oil and molten $\mathrm{Pb}$-Bi was relocated to the vessel bottom, where it was discharged from the hole together with silicone oil; (3) In the middle of the experiment, the hole at the vessel bottom was clogged by solidified $\mathrm{Pb}-\mathrm{Bi}$; (4) Then discharge of silicone oil was stopped and ablation of the vessel wall proceeded rapidly; (5) Finally, liquid in the vessel was discharged by penetrating the sidewall whose location was a little lower than liquid surface level.

Although the scale and materials used in the above experiment are different from those of RPV ablation by corium in a severe accident, it is expected that, the experiment captures the governing phenomena (convection, stratification, phase change and heat transfer) and provides key information needed to investigate capability of MPS method for the analysis.

\subsection{Analysis Conditions}

Fig. 2 shows calculation geometry of the MPS simulation. Table 2 shows the calculation parameters. In the analysis, the hole at the vessel bottom is neglected, because the hole at the vessel bottom is clogged by solidified $\mathrm{Pb}-\mathrm{Bi}$ in the middle of the experiment. In addition, silicone oil was in the vessel

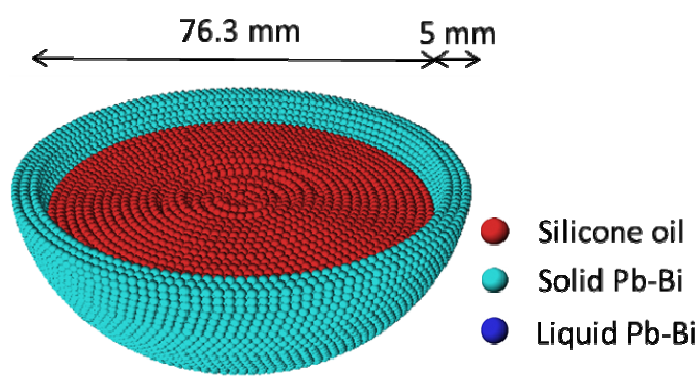

Fig. 2 Calculation geometry of the MPS. 
Table 2 Calculation parameters.

\begin{tabular}{lll}
\hline Material & $\mathrm{Pb}-\mathrm{Bi}$ & Silicone oil \\
\hline Density $\left(\mathrm{kg} / \mathrm{m}^{3}\right)$ & $10,050.0$ & 969.0 \\
Specific heat $(\mathrm{J} /(\mathrm{kg} \cdot \mathrm{K}))$ & 147 & $1,510-1,680$ (temperature dependent) \\
Thermal conductivity $(\mathrm{W} /(\mathrm{m} \cdot \mathrm{K}))$ & 13.8 & 0.16 \\
Kinematic viscosity coefficient $\left(\mathrm{m}^{2} / \mathrm{s}\right)$ & $1.29 \times 10^{-7}$ & $2.00 \times 10^{-4}$ at $25^{\circ} \mathrm{C}$ (temperature dependent) \\
Initial temperature $\left({ }^{\circ} \mathrm{C}\right)$ & 26 & 300 \\
Melting temperature $\left({ }^{\circ} \mathrm{C}\right)$ & 123.85 & - \\
\hline
\end{tabular}

beforehand in the analysis, whereas it was poured into the vessel and it took $1.6 \mathrm{~s}$ in the experiment. The current MPS simulation was focused on vessel ablation by silicone oil after it was filled into the vessel. Investigations on effects of poring and discharge of silicone oil will be for future studies.

According to Table 1, the experiment was conducted at room temperature, so heat removal by the air was considered in the analysis. The empirical formulas about natural convection heat transfer of a hemisphere and a plate are used [17] to model heat removal by the air. The empirical formula of a hemisphere is used for outer surface of the vessel and that of plate is used for top surface of liquid in the vessel. These are expressed by Nusselt number $(\mathrm{Nu})$ as follows.

For outer surface of the vessel:

$$
N u=2+0.760 C_{1} R a^{m}
$$

where

$$
\begin{gathered}
C_{1}=\frac{3}{4}\left(\frac{P r}{2.4+4.9 \sqrt{P r}+5 P r}\right)^{1 / 4} \\
m=\frac{1}{4}+\frac{1}{4+8.2 R a^{0.095}} \\
\operatorname{Pr}=0.717 \\
R a=3.81
\end{gathered}
$$

For top surface of silicone oil:

$$
N u=3.053 \times 10^{-2} R a^{2 / 5}
$$

where

$$
R a=1.23 \times 10^{3}
$$

where, $\operatorname{Pr}$ is Prandtl number of the air and $R a$ is Rayleigh number of the air. Then, $N u$ is expressed as follows:

$$
N u=\frac{h d}{k}
$$

where

$$
k=0.02614(\mathrm{~W} /(\mathrm{m} \cdot \mathrm{K}))
$$

where, $d$ is the diameter of the hemisphere, $k$ is thermal conductivity of the air, $h$ is heat transfer coefficient of the air. According to Eqs. (14)-(16), $h$ is obtained. Then Newton's law of cooling is expressed as follows:

$$
q=h\left(T_{\omega}-T_{\infty}\right)
$$

where, $q$ is heat flux, $T_{\omega}$ is the surface temperature, $T_{\infty}$ is the external temperature. Hence, $q$ is the amount of heat removal by the air.

In analysis of the reference case, the particle size of $1.667 \mathrm{~mm}$ and the corresponding total particle number of 29,007 is used. Some results of sensitivity study on the particle size of $2.5 \mathrm{~mm}$ (corresponding total particle number of 8,582) are also presented in Section 3.3.

\subsection{Results and Discussions}

3.3.1 Qualitative Discussions with Reference Case Analysis Results

Fig. 3 shows the cross-sectional view of the liquid discharge from the failed sidewall of the vessel in the analysis. As shown in Fig. 3, the ablation initiates at the wall slightly below the top surface of silicone oil and molten $\mathrm{Pb}-\mathrm{Bi}$ relocates to the bottom of the vessel. The ablation continues until the entire wall thickness has been ablated at around $74.1 \mathrm{~s}$ and silicone oil is discharged from the so called "fish mouth" of the failed wall. Fig. 4 shows side view of liquid discharge from the failed sidewall of the vessel in the experiment at $70 \mathrm{~s}$. The two arrows indicate the failed regions (the red arrow pointing at the leakage from the fish mouth-like hole and the blue arrow pointing at the small leakage) in Fig. 4. The analysis well reproduced the liquid discharged from the fish mouth-like hole 


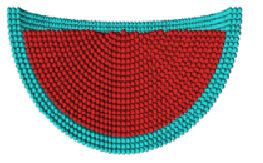

Os

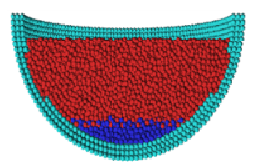

$15 \mathrm{~s}$

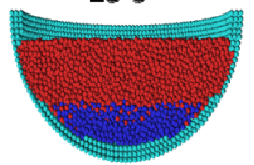

$40 \mathrm{~s}$

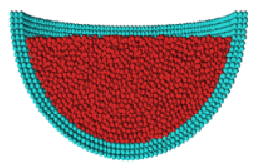

$5 \mathrm{~s}$

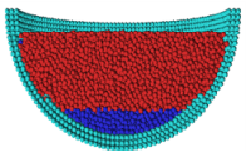

$20 \mathrm{~s}$

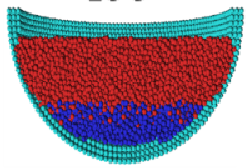

$50 \mathrm{~s}$

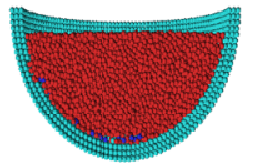

$11 \mathrm{~s}$

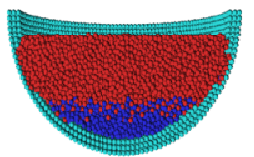

$30 \mathrm{~s}$

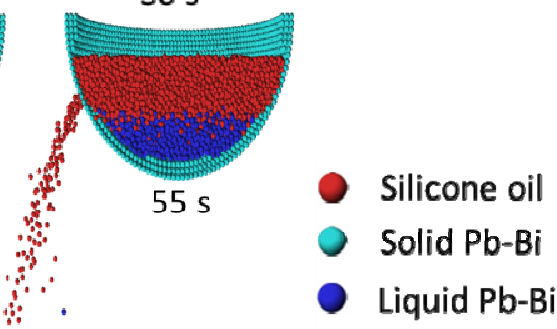

Fig. 3 Cross-sectional view of the liquid discharge from the failed sidewall of the vessel in the analysis.

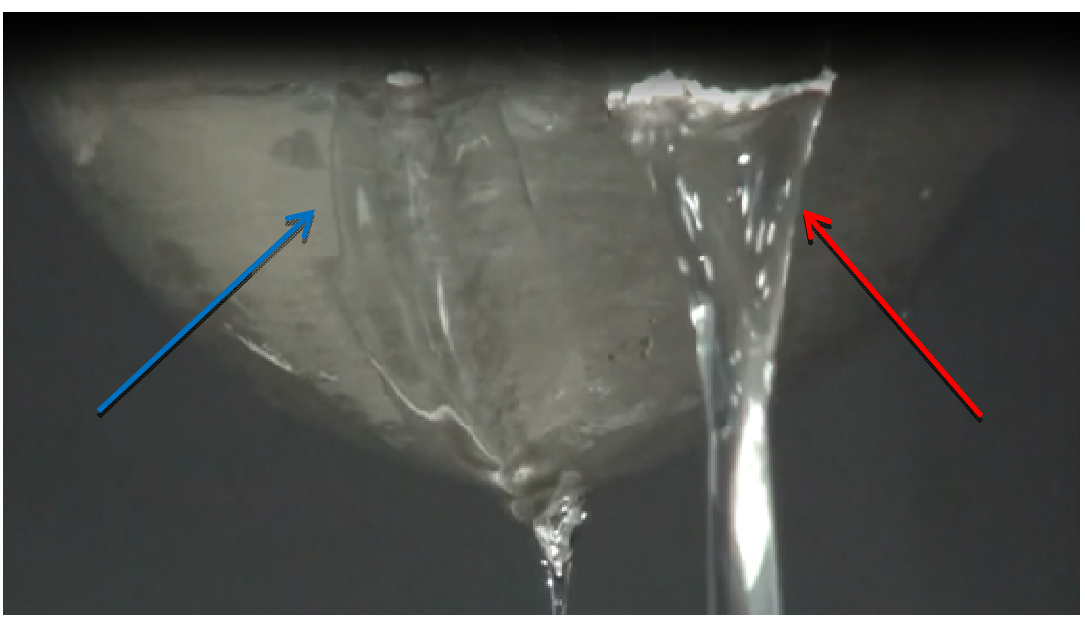

Fig. 4 Side view of the liquid discharge from the failed sidewall of the vessel in the experiment $(70 \mathrm{~s})$.

(ablated side wall). Predicted position of the failure also agrees with the experiment qualitatively. However, the small leakage observed in the experiment (indicated by the blue arrow in Fig. 4), was not reproduced.

Fig. 5 shows temperature distribution of liquid in the vessel in the analysis. Initially, heat of the silicone oil at the boundaries was transferred to the vessel wall and the air. As time proceeded, convection of the silicone oil particles was induced due to the temperature distribution. As the result, the temperature distribution of the silicone oil was stratified and the layer of high temperature was formed on the upper part of the vessel. The stratified temperature distribution was also influenced by the convection after the $\mathrm{Pb}-\mathrm{Bi}$ wall particles started to melt and relocate to the bottom of the vessel by density difference. It is considered that, this temperature stratification phenomenon and heat concentration in the upper part of the vessel were responsible for liquid discharge from the vessel side wall whose location was a little lower than liquid surface level.

Fig. 6 shows top view of the ablation of the $\mathrm{Pb}-\mathrm{Bi}$ vessel in the analysis. In Fig. 6, the particles representing silicone oil are not depicted to better show ablation of the vessel. As explained earlier, ablation initiates at 


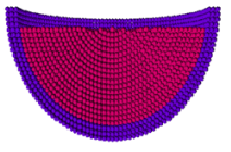

$\mathrm{OS}$

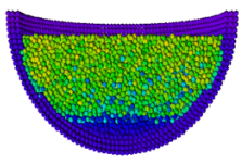

$15 \mathrm{~s}$

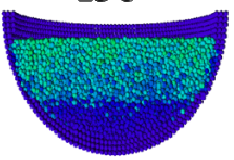

$40 \mathrm{~s}$

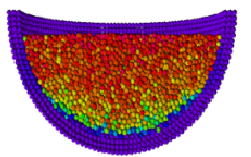

$5 \mathrm{~s}$

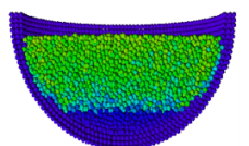

$20 \mathrm{~s}$

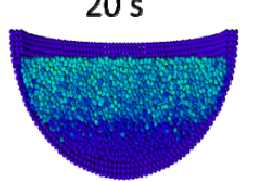

$50 \mathrm{~s}$

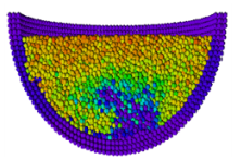

$11 \mathrm{~s}$

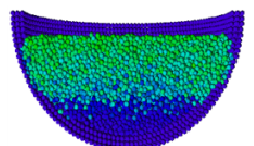

$30 \mathrm{~s}$

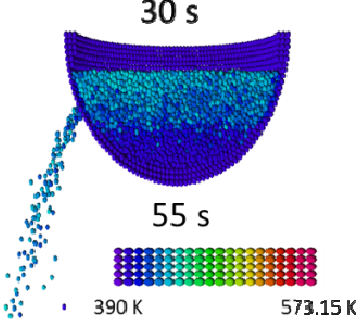

Fig. 5 Temperature distribution of the liquid in the vessel in the analysis.

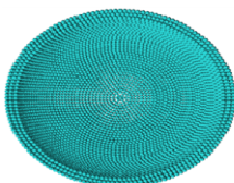

O s

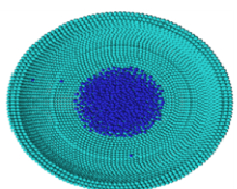

$15 \mathrm{~s}$

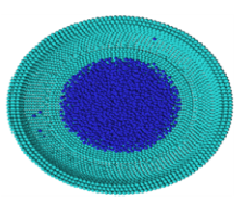

$40 \mathrm{~s}$

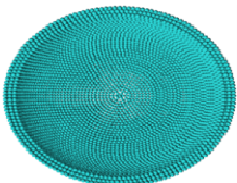

$5 \mathrm{~s}$

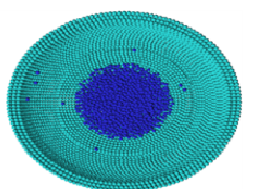

$20 \mathrm{~s}$

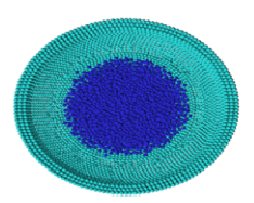

$50 \mathrm{~s}$

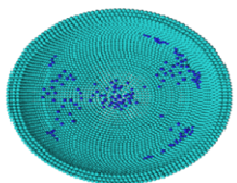

$11 \mathrm{~s}$

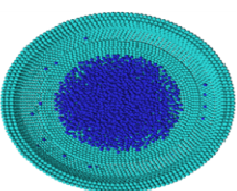

$30 \mathrm{~s}$

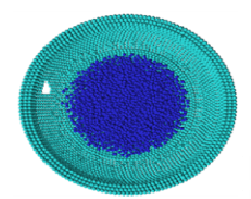

$55 \mathrm{~s}$
Solid Pb-Bi

Liquid Pb-Bi

Fig. 6 Top view of the ablation of the $\mathrm{Pb}-\mathrm{Bi}$ vessel in the analysis.

the upper part of the vessel and molten $\mathrm{Pb}$-Bi relocates to the bottom of the vessel. Fig. 7 shows the picture of vessel wall ablation in the experiment. Since silicone oil is transparent, relocation of molten $\mathrm{Pb}$ - $\mathrm{Bi}$ could be visually observed. The arrow in Fig. 7 indicates ablation start point. In the experiment, ablation was also initiated in the upper part of the vessel and molten $\mathrm{Pb}-\mathrm{Bi}$ was relocated and accumulated at the vessel bottom.

As for these qualitative comparisons, the analysis results of the MPS method well reproduced the experimental results. Noticeably, it can be seen for both the experiment and simulation that, locations of generation of the molten $\mathrm{Pb}-\mathrm{Bi}$ droplets are distributed unsymmetrically. It is considered that, these asymmetries arise from imperfection of the vessel for both the experiment and simulation. In the experiment, there is always some asymmetry due to imperfection in manufacturing of the vessel and alignment of the experiment. Similarly, in the MPS simulation, the calculation geometry of the MPS method is not perfectly symmetrical. The particle arrangement of the vessel wall is not uniform. This is expected to be the reason for the unsymmetrical results in the analysis. 


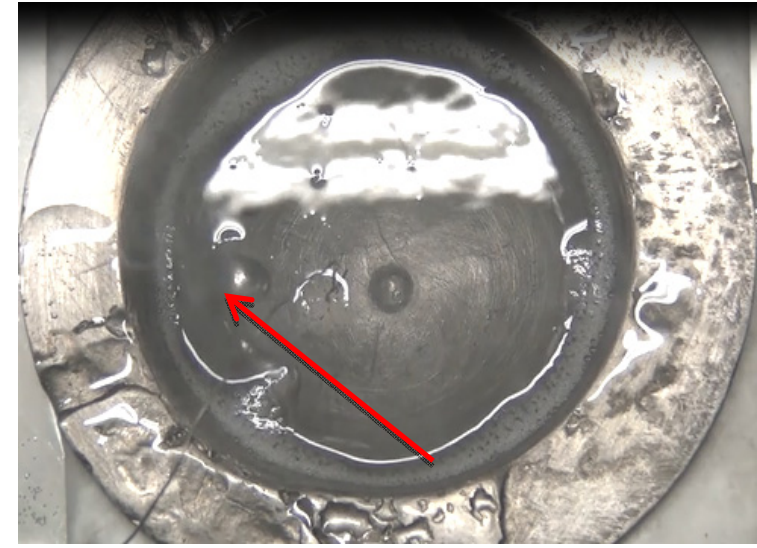

(a) $25 \mathrm{~s}$

Fig. 7 Picture of vessel wall ablation in the experiment.

3.3.2 Quantitative Discussions and Sensitivity Analyses

In the previous section, the reference analysis results were compared with the experimental results qualitatively. In Chapter 3.3.2, some quantitative comparisons are made with addition of sensitivity analysis results using larger calculation particle size of $2.5 \mathrm{~mm}$ and smaller calculation particle size of $1.25 \mathrm{~mm}$ (particle size of the reference case is $1.667 \mathrm{~mm}$ ). Particle size in MPS method indicates resolution of discretization of the simulation and should be small enough to capture governing phenomena of the interest. On the other hand, smaller particle size leads to larger number of particles required to cover the calculation domain, which increases calculation cost. It should be noted that, there was no qualitative difference in the analytical results by changing the particle size. However, significant differences were identified quantitatively as described in the followings.

Table 3 shows comparison of the start time of vessel wall ablation and liquid discharge from the vessel between the analysis and the experiment. In the experiment, start time of vessel wall ablation (18.5 s) is defined when the first $\mathrm{Pb}-\mathrm{Bi}$ liquid droplet is visually observed. In contrast, the corresponding start time in the simulation is when the first $\mathrm{Pb}-\mathrm{Bi}$ wall particle is molten to liquid $\mathrm{Pb}-\mathrm{Bi}$ particle. As described in Section 2.2, a wall particle turns to a

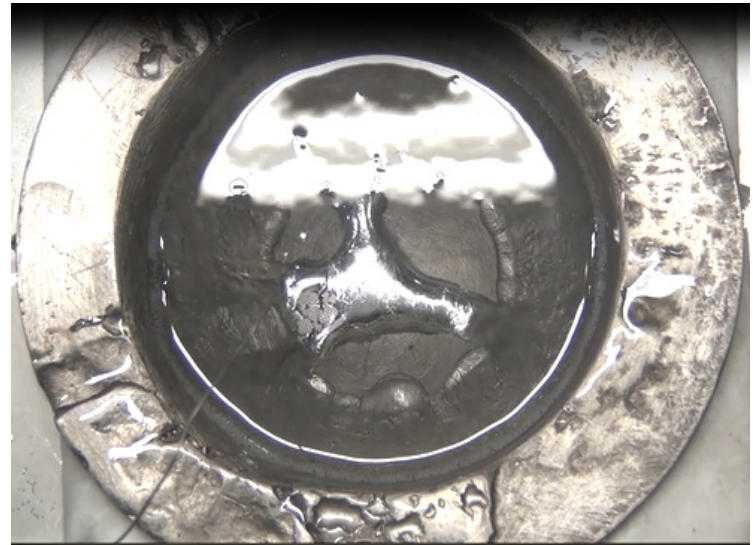

(b) $60 \mathrm{~s}$

liquid particle when enthalpy of the particle exceeds the melting point. The predicted start time of the vessel wall ablation with particle size of $1.667 \mathrm{~mm}$ and $1.25 \mathrm{~mm}$ is much earlier than that with $2.5 \mathrm{~mm}$. It is because enthalpy needed for ablation of one $\mathrm{Pb}-\mathrm{Bi}$ particle decreases as particle size is reduced. The MPS results with smaller particle size of $1.667 \mathrm{~mm}(10.6 \mathrm{~s})$ and $1.25 \mathrm{~mm}(6.5 \mathrm{~s})$ indicates that, MPS predictions are earlier than the experimentally observed start time (18.5 s) of the vessel wall ablation. However, it should be noted that, in the experiment, some small ablation may have occurred earlier than the recorded time of $18.5 \mathrm{~s}$, but may have not been noticed by visual observation. Hence, there may be large uncertainty regarding the experimentally determined start time of ablation. It may be for a future study to consider other ways of comparisons because the start time of vessel wall ablation depends on the particle size. For example, comparison of certain area (or volume) of wall ablation may be considered.

Table 3 also shows comparison of start time of discharge of the liquid from the vessel from the fish mouth of the failed vessel wall. Reduction in predicted discharge time with smaller particle size indicates that, more investigation may be necessary regarding influence of particle size in the MPS method. The MPS results with smaller particle size of $1.667 \mathrm{~mm}(52.4 \mathrm{~s})$ and $1.25 \mathrm{~mm}(42.9 \mathrm{~s})$ indicate that, MPS predictions are earlier than the experimentally observed start time 
Table 3 Comparison of the start time of vessel wall ablation and liquid discharge from the vessel between the analysis and the experiment.

\begin{tabular}{lllll}
\hline \multirow{2}{*}{ Start time } & \multirow{2}{*}{ Experiment } & \multicolumn{3}{c}{ MPS analysis } \\
\cline { 3 - 5 } & & Particle size: $2.5 \mathrm{~mm}$ & Particle size: $1.667 \mathrm{~mm}$ & Particle size: $1.25 \mathrm{~mm}$ \\
\hline Vessel wall ablation (s) & 18.5 & 24.0 & 10.6 & 6.5 \\
Discharge from the vessel (s) & 68.6 & 74.1 & 52.4 & 42.9 \\
\hline
\end{tabular}

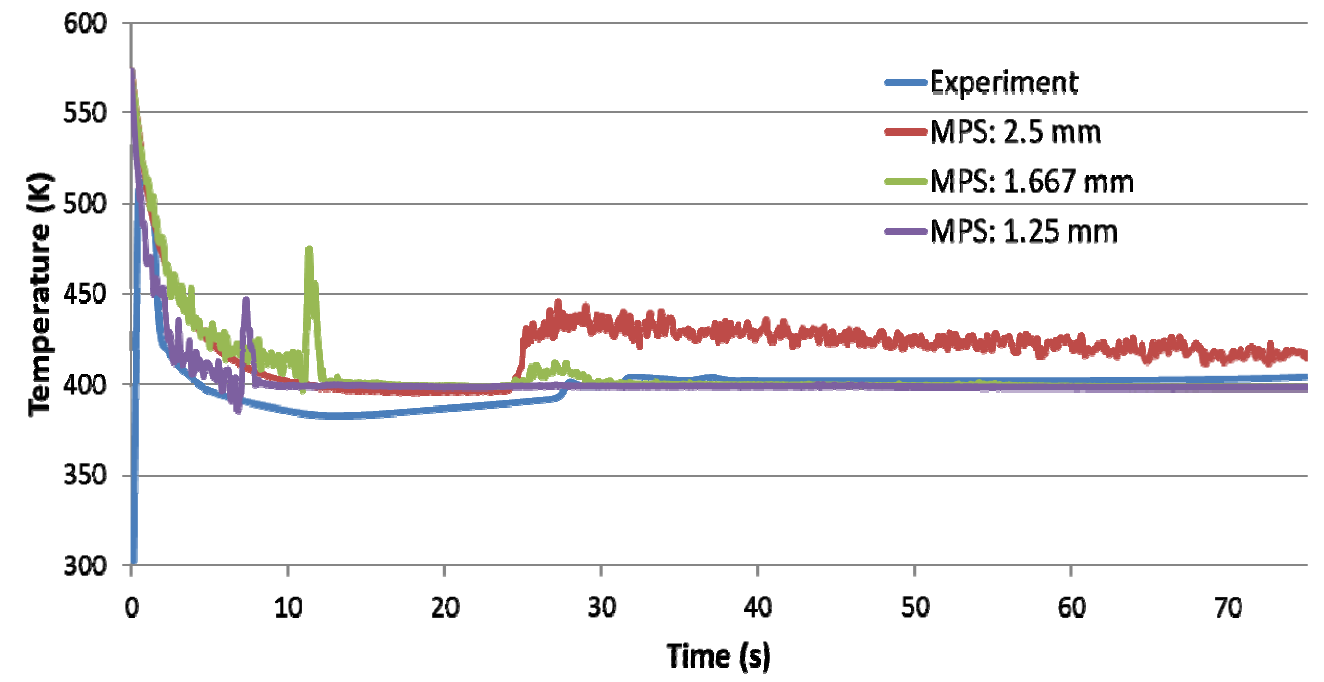

Fig. 8 Comparison of liquid temperature at the vessel bottom between the analysis and the experiment.

(68.6 s) of the discharge. However, particle size needs to be sufficiently small to accurately model heat transfer, convection and phase change, which affect the discharge start time.

Fig. 8 shows the comparison of liquid temperature at the vessel bottom between the analysis and the experiment. The "Experiment" indicates the temperature measured by a thermocouple, which was attached at the bottom inner surface of the vessel wall. In contrast, the calculated temperatures are the average temperatures of liquid particles at the bottom of the vessel, corresponding to location of the thermocouple.

The analysis with particle size of $2.5 \mathrm{~mm}$ over-predicts the measured liquid temperature through the entire duration, while that with particle size of $1.667 \mathrm{~mm}$ and $1.25 \mathrm{~mm}$ agrees well with the measurements after around $30 \mathrm{~s}$. However, all analytical results are a little higher than the measurements up to around $30 \mathrm{~s}$. As described in Section 3.2, there are some differences between the experimental and analytical conditions, which may be responsible for the difference in the early phase. In the simulation, pouring of silicone oil at the beginning and discharge of silicone oil until the hole was clogged at $32.6 \mathrm{~s}$ was neglected. These differences may have led to the over-predictions, because loss of enthalpy of silicone oil to the air (during pouring) and by discharge through the hole is not considered in the analysis. Another possibility is that, the MPS heat transfer model is sufficiently accurate to predict the liquid temperature distribution under pseudo-steady state condition observed in the late phase of the experiment, while it needs further modifications to accurately predict the temperature distribution during rapid transient as observed in the first $30 \mathrm{~s}$ of the experiment.

The trends of the calculated temperatures with particle size of $1.667 \mathrm{~mm}$ and $1.25 \mathrm{~mm}$ are almost similar. Namely, there is a sharp increase in the calculated temperature at around $10 \mathrm{~s}$, and that after around $30 \mathrm{~s}$, the calculated temperatures are almost the same and agree well with the measurements. As the 
trend of result with particle size of $2.5 \mathrm{~mm}$ is significantly different from the others, particle size of $2.5 \mathrm{~mm}$ might be too large for analysis. On the other hand, as the trends of results with particle size of $1.667 \mathrm{~mm}$ and $1.25 \mathrm{~mm}$ are almost similar, particle size of $1.667 \mathrm{~mm}$ might be small enough for analysis.

In the analysis with particle size of $1.667 \mathrm{~mm}$ and $1.25 \mathrm{~mm}$, the calculated temperature with particle size of $1.25 \mathrm{~mm}$ sharply increases at around $7 \mathrm{~s}$ and that of $1.667 \mathrm{~mm}$ sharply increases at around $11 \mathrm{~s}$, which coincide with the corresponding start time of the vessel wall ablation (6.5 s and $10.6 \mathrm{~s})$. At this time, the simulation indicates promoted downward flow of high temperature silicone oil from the top surface by relocation of molten $\mathrm{Pb}-\mathrm{Bi}$ particle. This may cause sharp increase in the calculated temperature in the analysis. However, such increase of the temperature is not found in the experiment. More quantitative investigations may be necessary to identify the responsible mechanisms.

\section{Conclusions}

In the scope of validating MPS method for predicting ablation of RPV in severe accident of a nuclear reactor, the $\mathrm{Pb}-\mathrm{Bi}$ ablation experiment was simulated with the MPS method. The MPS method incorporating phase changes (solidification, melting and ablation of metals), convection, stratification and heat transfer models well predicted the experiment qualitatively with respect to location of the vessel wall failure. However, some quantitative discrepancies have been identified with respect to temperature of the confined liquid and time of the vessel failure. The results indicated that, more investigations are necessary with respect to the above mentioned models as well as influence of the particle size.

\section{Acknowledgments}

The MPS code of the present study was developed based on MPS-SW-MAIN-Ver.2.0 which was kindly provided by Professor S. Koshizuka and Dr. K. Shibata of the University of Tokyo. A part of this study is the result of "mechanistic study of melt behavior in lower RPV head" carried out under the Initiatives for Atomic Energy Basic and Generic Strategic Research by the Ministry of Education, Culture, Sports, Science and Technology of Japan.

\section{References}

[1] Hodge, S. A. 1989. "BWR Reactor Vessel Bottom Head Failure Modes.” Oak Ridge National Lab. Accessed July 5 ,

2013. http://www.iaea.org/inis/collection/NCLCollectionStore/ Public/20/060/20060972.pdf.

[2] Park, R., Kang, K., Hong, S., Kim, S., and Song, J. 2012. "Corium Behavior in the Lower Plenum of the Reactor Vessel under IVR-ERVC Condition: Technical Issues." Nuclear Engineering and Technology 44 (3): 237-48.

[3] Humphries, L. L., Chu, T. Y., Bentz, J., Simpson, R., Hanks, C., Lu, W., Antoun, B., Robino, C., Puskar, J., and Mongabure, P. 2002. "OECD Lower Head Failure Project Final Report (Volume 1-Integral Experiments and Material Characterization." Sandia National Laboratories. Accessed June 7, 2015. https://www.oecd-nea.org/nsd/docs/2002/csni-r2002-27.p df.

[4] Sehgal, B. R., Nourgaliev, R. R., and Dinh, T. N. 2002. "Characterization of Heat Transfer Processes in a Melt Pool Convection and Vessel-Creep Experiment." Nuclear Engineering and Design 211 (2-3): 173-87.

[5] Gauntt, R., Kalinich, D., Cardoni, J., Phillips, J., Goldmann, A., Pickering, S., Francis, M., Robb, K., Ott, L., Wang, D., Smith, C., St.Gemain, S., Schwieder, D., and Phelan, C. 2012. "Fukushima Daiichi Accident Study." Sandia National Laboratories. Accessed June 12, 2015. https://fukushima.inl.gov/PDF/FukushimaDaiichiAcciden tStudy.pdf.

[6] Yang, R., and Rahn, F. 2013. "Fukushima Technical Evaluation: Phase 1-MAAP5 Analysis." Electric Power Research Institue. Accessed May 28, 2015. http://www.epri.com/abstracts/Pages/ProductAbstract.asp $\mathrm{x}$ ?ProductId $=000000000001025750$.

[7] Naitoh, M., Pellegrini, M., Mizouchi, H., Suzuki, H., and Okada, H. 2013. "Analysis of Accident Progression of Fukushima Daiichi Npps with SAMPSON Code." In Proceedings of the 23th International Conference on Nuclear Engineering (ICONE-21), 1-7.

[8] Gauntt, R. O., Cole, R. K., Erickson, C. M., Gido, R. G., Gasser, R. D., Rodriguez, S. B., Young, M. F., Ashbaugh, S., Leonard, M., and Hill, A. 2000. "MELCOR Computer Code Manuals: Primer and User's Guide Version 1.8.5 
(NUREG/CR-6119, Volume 1, Revision 2)." Sandia National Laboratories. Accessed July 15, 2015. http: //pbadupws.nrc.gov/docs/ML0101/ML010120456.pdf.

[9] Koshizuka, S., and Shibata, K. 2006. "MPS-SW-MAIN-Ver.2.0." Copyright Registration P8827-1, Tokyo.

[10] Kawahara, T., and Oka, Y. 2012. "Ex-Vessel Molten Core Solidification Behavior by Moving Particle Semi-Implicit Method.” Nuclear Science and Technology 49 (12): 1156-64.

[11] Chen, R., and Oka, Y. 2014, "Numerical Analysis of Freezing Controlled Penetration Behavior of the Molten Core Debris in an Instrument Tube with MPS.” Annals of Nuclear Energy 71 (September): 322-32.

[12] Li, X., and Oka, Y. 2014. "Numerical Simulation of the SURC-2 and SURC-4 MCCI Experiments by MPS Method." Annals of Nuclear Energy 73 (November): 46-52.
[13] Li, G., Oka, Y., Furuya, M., and and Kondo, M. 2013. "Experiments and MPS Analysis of Stratification Behavior of Two Immiscible Fluids." Nuclear Engineering and Design 265 (December): 210-21.

[14] Li, G., Oka, Y., and Furuya, M. 2014. "Experimental and Numerical Study of Stratification and Solidification/Melting Behaviors." Nuclear Engineering and Design 272 (June): 109-17.

[15] Masahiro, F., and Oka, Y. 2015. "Ablation and Melting Relocation of Hemisphere Vessel Due To Natural Convection." In Proceedings of the 23th International Conference on Nuclear Engineering (ICONE-23), 1-4.

[16] Li, G. 2015. "Study on Melt Behavior in a BWR Lower Head by MPS Method and MELCOR Code." Doctor thesis, Waseda University.

[17] The Japan Society of Mechanical Engineers. 1986. JSME Data Book: Heat Transfer 4th Edition. Tokyo: The Japan Society of Mechanical Engineers, 68-71. 